\title{
Evaluation of Coagglutination Test Kit for Red Sea Bream Iridovirus
}

\author{
Surya Amanu ${ }^{1}$, Achmad Bahtiar Rifai ${ }^{2}$, Lina Yulianah² and Pramudya Dwiwahyu ${ }^{2}$ \\ 1. Department of Microbiology, Faculty of Veterinary Medicine, Gadjah Mada University, Yogyakarta 560862, Indonesia \\ 2. Fish Quarantine and Inspection Agency, Batam 29464, Indonesia
}

\begin{abstract}
Coagulation test, in principle, is an immunodiagnostics technique, in which immunoglobuline G of antibody is bound to protein A from Staphylococcus aureus. The aim of study is to develop a rapid test kit for detecting iridovirus infection in fish. Method was summarized as follows: (1) vaccine of iridovirus was injected to rabbit four times with a dosage as $0.5 \mathrm{~mL}, 1 \mathrm{~mL}, 2 \mathrm{~mL}$, $3 \mathrm{~mL}$ each week. Serum was collected at the fifth week as a coagglutination test kit; (2) through the positif polymerase chain reaction (PCR) test, the kidney and spleen sample infected with iridovirus are homogenized by using the phosphate buffered saline (PBS) solution of $\mathrm{pH} 7.2$ with ratio 1:2 (W/V); (3) the supernatant material is collected after centrifugation at 8,000 rpm for $15 \mathrm{~min}$; (4) filtrate/supernatant from sample was dropped on a slide an added with coagglutination test kit with the same volume (1:1); (5) the agglutination observation is done after the 30,60 and 90 min incubate at room temperature. The coagglutination test gave positive result in $25 \%$ of the test samples.
\end{abstract}

Key words: Diagnostics, coagglutination, test kit, iridovirus.

\section{Introduction}

Immunodiagnostic test using coagglutination with antibody-sensitised Staphylococcus has been recognized for its ability to detect bacterial and viral pathogen in fish. The method is proven to have many advantages for its characteristics, such as simple, fast, accurate and inexpensive especially for field application [1-6].

This test does not require sophisticated laboratory equipment, but its value has been overshadowed by more expensive tools, such as polymerase chain reaction (PCR) or flouresent antibody technique (FAT). On the other side, the test result needs less time rather than traditional/conventional diagnostic test.

Coagglutination test is an immunodiagnostic technique, in which immunoglobulin $\mathrm{G}$ of antibody is bound to protein A from Staphylococcus aureus. This test can detect the presence of specific antigen in

Corresponding author: Kurniasih, professor, research fields: fish and prawn disease. kidney and spleen or affected tissue of fish.

Red sea bream iridovirus (RSIV) is the genus member that firstly was reported as the cause of serious illness especially for the marine culture fish species in East and South East Asia. Iridovirus is one of virus known as the main cause of grouperfish death. The infection of iridovirus for the grouper caused the grouper sleepy disease iridovirus (GSDIV). This infection of virus caused the mass death only in few days or weeks, the infected fish showed clinically symptom, such as abnormal swimming, lethargie and mortality sometimes reached $50 \%-90 \%$ within two months [7]. Histopathological studies of the affected fish showed enlarged basophilic cells in the gill, kidney, heart, spleen and liver [7, 8].

The aim of study was to develop a rapid test kit for detecting iridovirus infection in fish from Indonesia.

\section{Materials and Methods}

\subsection{Immunoglobulin Preparation}

Separation of immunoglobulin is done by 
ammonium sulfate precipitation method [5, 9]. Equal amount of immune serum was added with 50\% ammonium sulfate ( $\mathrm{pH}$ 8.0) slowly while stirring by stirrer for $30 \mathrm{~min}$. The suspension was centrifuged (6,000 rpm, $30 \mathrm{~min}$ ), the supernatant was discarded and the pellet was resuspended again in physiological saline to initial volume and then re-precipitated using ammonium sulfate. Repeat this process up to three times. Precipitate/final pellet was re-dissolved in phosphate buffered saline (PBS, $\mathrm{pH}$ 7.2) to a number of initial volume and placed in a dialysis membrane. Aliquot is washed by immersing in dialysis membrane PBS solution for $48 \mathrm{~h}$, with replacement of PBS solution every $12 \mathrm{~h}$.

\subsection{Preparation of Protein A}

S. aureus isolates used is collected from the Faculty of Veterinary Medicine, University of Gadjah Mada. Isolates was propagated in tryptic soy agar (TSA) plate, harvested and centrifuged at 3,000 rpm for 15 min three times. The final pellet was added $2 \%$ formalin and left overnight at room temperature. Aliquot then was heated at a temperature of $80{ }^{\circ} \mathrm{C}$ water bath for 15 min and immediately cooled in the refrigerator. Final suspension was centrifuged at 3,000 rpm for $15 \mathrm{~min}$ and the pellet was added PBS 1:10 (w/v) [5].

\subsection{Coupling of Immunoglobulin $G$ and Protein A}

Suspension of $S$. aureus and immunoglobulin G with volume of $1: 1$ is homogenized. After incubation for $2 \mathrm{~h}$ at room temperature, the suspension was centrifuged at 1,500 rpm for $20 \mathrm{~min}$. Supernatant was discarded, PBS was added to return to the initial suspension volume and was used as a reagent for the coagglutination test.

\subsection{Positive Sample}

The positive sample of iridovirus was collected from silver pompano (Trachinotus blochii) through the PCR test from Batam Island. That fish will be necropsied by taking some organs/specimen, such as kidney and spleen, meanwhile the negative control is using Nucleus-free water.

\subsection{Coagglutinatiom Test Procedure}

Coagglutination test procedure was carried out using method described by Kimura and Yoshimizu [2]. The organ sample was homogenized using the physiological saline solution with ratio of 1:1, 1:2 and 1:4 (w/v) and then centrifuged at 8,000 rpm for 15 min. Filtrate/supernatant from sample was dropped on a slide and added with coaglutination test kit with the same volume (1:1). The agglutination observation is done after the 30, 60 and 90 min incubation [2, 3, 10]. The positive control uses vaccine killed Aqua Irido Vaccine product from Intervet International BV and the negative control uses Nucleus-free water .

\section{Results and Discussion}

Serum anti to iridovirus is bound to protein A from Staphylococcus aureus which can large size, when the S. aureus-IgG coupling is bond to iridovirus antigen, a large complete is formed in spite of the amount of antigen, so it can be used to detect iridovirus of the organ without the need any special apparatus and can be seen with the naked eyes. Identification of iridovirus infection in fish using co-agglutination test takes only 30-60 min. The result showed that the test kit is able to detect iridovirus from organ samples in infected fish, faster, simpler and more economical than conventional biochemical.

The positive result was marked by little sand around the suspension and can be seen with eyes or by the magnifying glass. For the negative result, the mixture suspension formed is a liquid and there is no agglomeration like sand (Fig. 1). This is happened because of the formation of agglutinin agglutinin $S$. Aureus than immunoglobulin complex with antigen from iridovirus. Antigen collected from spleen and kidney of fish which was infected by iridovirus showed little sand around the suspension (Fig. 2). 


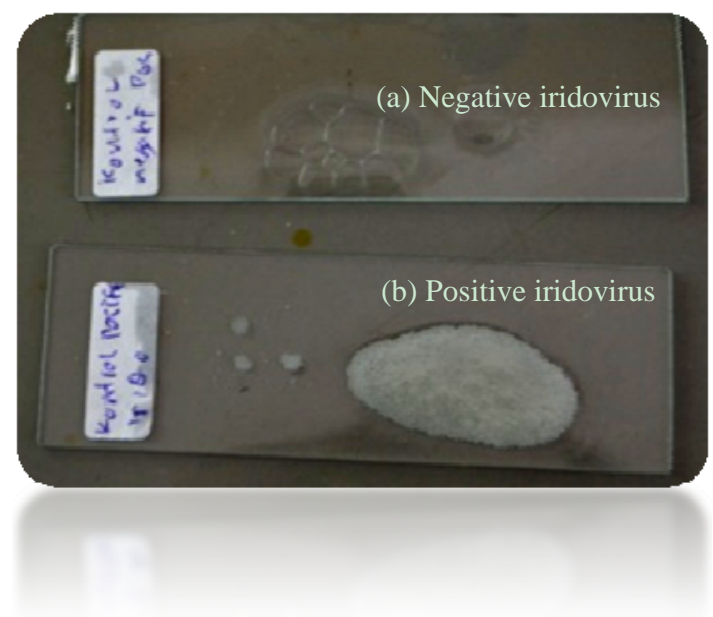

Fig. 1 Positive control iridovirus and negative control iridovirus.

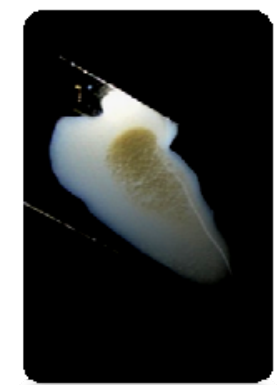

(a) Spleen

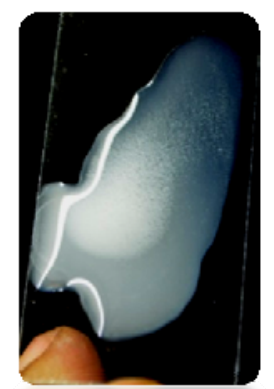

(b) Kidney
Fig. 2 Spleen and kidney positive iridovirus.

In the present study, coagglutination test gave positive results in $25 \%$ of the test samples and Rotalex test gave positive results in $20 \%$ of the cases [4], proving that coagglutination is more sensitive than Rotalex test. In control group, coagglutination test was positive only in $2.5 \%$ of cases, showing that coagglutination test correlates well with Rotalex test. The Rotalex kits are to be imported and are costly. Therefore, coagglutination test is a good substitute for mass screening and quick diagnosis of rotavirus infection [3].

\section{Conclusions}

The application of the coagglutination test using Staphylococci specifically sensitized with protein A
Staphylococcus aureus was able to detect iridovirus directly from the spleen and kidney infected organ of fish.

The most importance of the co-agglutination test in the diagnostic of fish disease would be to detect the specific viral antigen in the organ or tissue of infected fish.

Coagglutination method is proven to have many advantages for its characteristics, such as simple, fast, accurate and in expensive especially for field applications.

\section{References}

[1] Bootland, L. M., and leong, J. A. 1992. "Staphylococcal Coagglutination, a Rapid Method of Identifiying Infectious Hematopoietic Necrosis Virus.” Applied and Enviromental Microbiology 58 (1): 6-13.

[2] Kimura, T., and Yoshomizu, M. 1983. "Coagglutination Test with Antibody-Sensitized Staphylococci for Rapid and Simple Serological Diagnostic of Fish Furunculosis.” Fish Pathology 17 (4): 259-62.

[3] Kimura, T., and Yoshimizu, M. 1984. "Coagglutination Test with Antibody-Sensitized Staphylococci for Rapid Serological Identification of Rough Strains of Aeromonas salmonicida." Nippon Suisan Gakkaishi 50 (3): 439-42.

[4] Mathur, M. S., and Bhave, G. G. 1993. "Comparative Evaluation of Coagglutination and Latex Agglutination Test (Rotalex Kit) for Detection of Rota Virus.” $J$. Postgrad. Med. 39 (3): 130-1.

[5] Saharia, P. K., and Prasad, K. P. 2001. "Development of Coagglutination Kit for the Diagnosis of Pseudomonas fluorescens Infection in Fishes.” Asian Fisheries Science 14 (3): 293-300.

[6] Taksdal, T., and Thoreed, K. 1999. "Evaluation of a Rapid Coagglutination (COA) Test for the Detection of Infectious Pancreatic Necrosis Virus in Tissue Sample of Atlantic Salmon (Salmo salar L.).” Journal of Fish Disease 22 (2): 117-24.

[7] Wang, C. S., Shih, H. H., Ku, C. C., and Chen, S. N. 2003. "Studies on Epizootic Iridovirus Infection among Red Sea Bream, Pagrus major (Temminck \& Schlegel), Cultured in Taiwan.” Journal of Fish Disease 26 (3): 127-33.

[8] Kurita, J., and Nakajima, K. 2012. "Megalocytiviruses.” Viruses 4 (4): 521-38.

[9] Garvey, J. S., Crener, N. E., and Sussdorf, D. H. 1977. Methods in Immunology: A Laboratory Test for 
Instruction and Research, 3rd ed.. New York: W.A. Benjamin Inc. Publisher.

[10] Yoshimizu, M., and Kimura, T. A. 1985. “Coagglutination
Test with Antibody-Sensitized Staphylococci for Rapid and Simple Diagnosis of Bacterial and Viral Diseases of Fish.” Fish Pathology 20 (2-3): 243-61. 